\title{
Today's medical education, its deviations and weaknesses
}

Medicine calls for continuous learning, starting from college education and then goes on along the entire professional practice. This is a generalized concept, so instead of discussing how long should education go on, it is necessary to make a profound reflection on how today's medical education accomplishes the goals or principles of medicine and to what extent it meets community needs.

Medical learning entails complexity, not because it may be tougher than other disciplines, but because it is focused on human beings, the most complex objects of the whole world. As a result, medical education requires a delicate balance of contents offered to students both at college and post-graduate curricula.

In my opinion, such balance has been disrupted, especially since the 1950s, and this is one of the main reasons for today's weak education systems, which undoubtedly have had a harmful influence on health care.

What do I mean by balance? First of all, everything we do in our daily lives should have a balance, which is represented by an attitude that encompasses harmony, humility, impartiality, and good judgment in all of our actions and opinions, among other attributes.

Today's medical education, in general, is far from being adequate because of different factors that have resulted in deviations or errors in education curricula which have excluded, separated or underestimated the fundamental aspects of medicine in relation to human sciences.

Let's first consider graduate teaching processes. The traditional structure of education at schools of medicine makes a marked distinction between a long common basic course and a shorter clinical course, which are not usually integrated. This has been detrimental to the training of future doctors by focusing on teaching medicine as if it were merely related to biological science, and nothing could be further from the truth.

In addition to it being centered on biology, several biases are present in the study of medicine, which overtly alter the balance I was talking about. Among others, one of the most common biases is assuming that molecular medicine will solve all or most of genetic conditions, although only very few cases have proven so to date. Even if it did happen in the future, the transfer of knowledge in the field of medicine should not be biased nor fragmented. However, medical education should be channeled with moderation and allow to neutrally point students to the current scope and long-term possibilities of medicine.

This example refers to the type of teaching most predominantly observed in graduate courses: incorrectly assuming that doctors will practice medicine by applying only their knowledge on biology. This is absolutely wrong and far from the reality of professional practice.

It is therefore vital to have the common basic course integrated with the essential aspects of the multiple disciplines included in clinical practice from the beginning because the main objective of medical university training is to introduce students to the professional practice. In this regard, learning objectives should be invariably geared towards all the qualities a doctor should have to be able to fully practice medicine by combining biology and human sciences.

Schools of medicine should clearly state the kind of doctors societies need; therefore and without exception, graduate courses should focus on the wider scope of the social characteristics of the population. Health care requires having an adequate knowledge of people's needs; only this way doctors will be able to comply with the ethical mandate of helping every society achieve the greatest well-being possible. Medicine cannot accomplish this by itself. It is therefore critical for medicine to harmoniously blend with other various disciplines, especially social sciences, art, literature, etc., as well as to provide guidance to governments responsible for improving their population's living conditions. Also from the beginning, it is necessary to promote a critical stance in future doctors, combined with the humility to embrace uncertainty, find potential mistakes, and learn from them.

All aspects herein mentioned will help students to develop a particular mindset, which has to be continuously stimulated in order to achieve an adequate clinical judgment and other irreplaceable skills necessary to become aware of the essence of medicine. There is no need to overwhelm students with theoretical courses; instead, through different strategies, they should be encouraged to ponder over them and make 
a strong approach to the discipline required to become a good doctor.

Unfortunately, all such conditions are not complied with in most schools of medicine across Argentina. At the Second National Meeting on Humanism in Medicine, celebrated last May, Prof. Elena Cisariuk presented a comprehensive review on the place reserved for human sciences in medical education programs. Of the 32 schools of medicine in Argentina, less than half approach the subject, some devote it just a few hours, and only one of the 32 schools offered a humanism program every year, including the rotating internship.

The outlook at post-graduate medical education is not promising either. Practically no program focuses on channeling practice through the principles I have mentioned here. Faced with a lack for desire and ability to reflect on the thinking process aimed at making a critical assessment of one's own actions, a gap develops which cannot be bridged by medical education.

As long as doctors assume that medical practice exclusively depends on the knowledge of biology and become dazzled by the overwhelming use of technology, they will lose, or not even develop, the necessary skills to make an adequate clinical judgment, and as a result, their mindset and attitudes towards patients will be compromised. It is common ground that there are currently a significant increase of doctors unable to make an accurate diagnosis through physical examination. For decades now, sophisticated imaging and lab tests have prevailed in consultations as the main, and sometimes only, resource for diagnosis.

Empathetically listening to patients and making an adequate physical examination seem to be "old fashioned."

Such attitudes are mostly a result of market conditions, but also of the poor quality of graduate medical education. For this reason, in several countries, including Argentina, schools of medicine are now making curricular modifications, reducing the length of common basic courses, and prioritizing programs focused on going back to the long lost practice of clinical examinations and learning at the patient's bedside.

Such factors will be of great help in medical training during and after internship years given that any solid knowledge gained as a student is more likely to last forever.

Post-graduate education should settle and promote that this is the most important aspect of medicine, while technology is a complement, not the other way round. This way doctors may improve diagnostic precision, benefit patients and reduce costs, which is essential for making medicine sustainable and more equitable.

One of the risks posed by technology is that it provides access to copious amounts of information, which are usually irrelevant and excessive of what a good doctor actually needs. A risk is that we might end up having, or probably already have, a generation of doctors who only have access to plentiful new information and assume that this makes them knowledgeable, but the reality is just the opposite.

It is essential for doctors to develop, from the beginning, a critical spirit that allows them to be skeptical of new information and concepts introduced as an undeniable truth. Gaining knowledge requires embracing uncertainty. It is also worth noting that in the field of medicine, being certain equals to having a "certificate for ignorance," as pointed out by Prof. Alberto Agrest in his memorable reflections.

Medical education is both intellectual and moral; it should train doctors to live surrounded by paradoxes and contradictions and make them develop the healthy habit of skepticism.

Patients expect doctors to have solid knowledge and experience, both critical skills for our profession, but also that we are capable of listening and we are with them by reassurance.

Such attitudes by themselves will convey that our biggest interest is to help them.

\section{Jose M. Ceriani Cernadas} Editor

http:/ /dx.doi.org/10.5546/aap.2014.eng.394 